Network Working Group

Request for Comments: 4580

Category: Standards Track

B. Volz

Cisco Systems, Inc.

June 2006

\title{
Dynamic Host Configuration Protocol for IPv6 (DHCPv6) Relay Agent Subscriber-ID Option
}

Status of This Memo

This document specifies an Internet standards track protocol for the Internet community, and requests discussion and suggestions for improvements. Please refer to the current edition of the "Internet Official Protocol standards" (STD 1) for the standardization state and status of this protocol. Distribution of this memo is unlimited.

Copyright Notice

Copyright (C) The Internet Society (2006) .

Abstract

This memo defines a new Relay Agent Subscriber-ID option for the Dynamic Host Configuration Protocol for IPv6 (DHCPv6). The option allows a DHCPv6 relay agent to associate a stable "Subscriber-ID" with DHCPv6 client messages in a way that is independent of the client and of the underlying physical network infrastructure.

Table of Contents

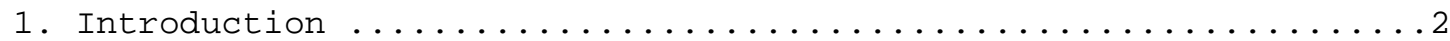

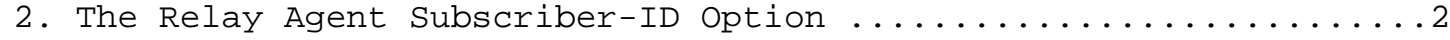

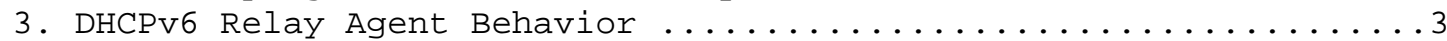

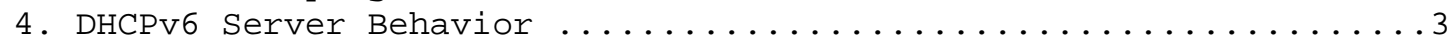

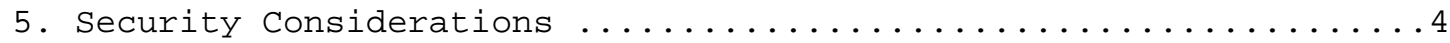

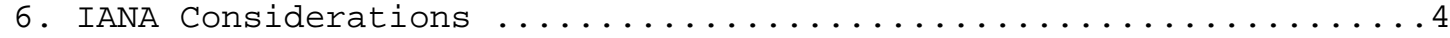

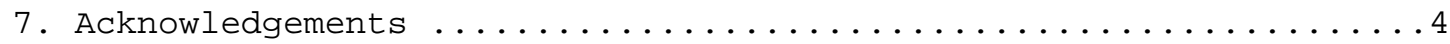

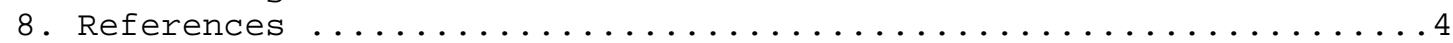

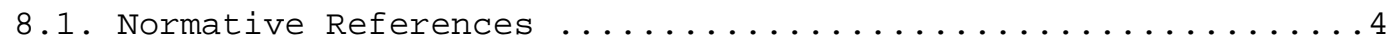

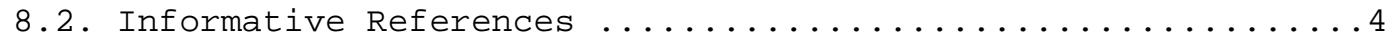




\section{Introduction}

DHCPV6 [1] provides IP addresses and configuration information for IPv6 clients. It includes a relay agent capability, in which processes within the network infrastructure receive multicast messages from clients and relay them to DHCPV6 servers. In some network environments, it will be useful for the relay agent to add information to the DHCPV6 message before relaying it.

The information that relay agents supply can also be used in the server's decision-making about the addresses, delegated prefixes [2], and configuration parameters that the client is to receive.

In many service-provider environments, it is believed to be desirable to associate some provider-specific information with clients' DHCPv6 messages that is independent of the physical network configuration and that the relay agent has learned through some means that is outside the scope of this memo.

2. The Relay Agent Subscriber-ID Option

In complex service provider environments, there is a need to connect a customer's DHCPv6 configuration with the customer's administrative information. The Relay Agent Subscriber-ID option carries a value that can be independent of the physical network configuration through which the subscriber is connected. This value complements, and might well be used in addition to, the network-based information. The "subscriber-id" assigned by the provider is intended to be stable as customers connect through different paths, and as network changes occur.

The subscriber-id information allows the service provider to assign/ activate subscriber-specific actions; e.g., assignment of specific IP addresses, prefixes, DNS configuration, trigger accounting, etc. This option is de-coupled from the access network's physical structure, so a subscriber that moves from one access-point to another, for example, would not require reconfiguration at the service provider's DHCPv6 servers.

The subscriber-id information is only intended for use within a single administrative domain and is only exchanged between the relay agents and DHCPv6 servers within that domain. Therefore, the format and encoding of the data in the option is not standardized, and this specification does not establish any semantic requirements on the data. This specification only defines the option for conveying this information from relay agents to DHCPv6 servers. 
However, as the DHCPv4 Subscriber-ID suboption [3] specifies Network Virtual Terminal (NVT) American Standard Code for Information Interchange (ASCII) [4] encoded data, in environments where both DHCPv4 [5] and DHCPv6 are being used, it may be beneficial to use that encoding.

The format of the DHCPv6 Relay Agent Subscriber-ID option is shown below:

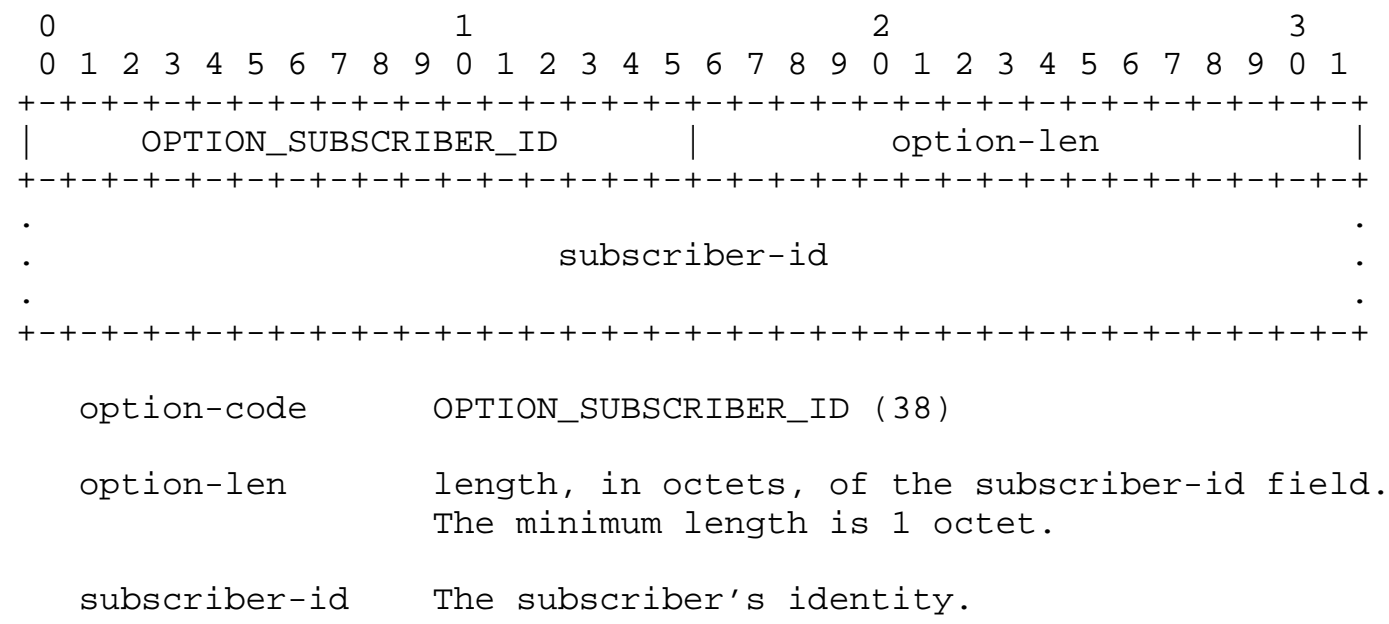

3. DHCPv6 Relay Agent Behavior

DHCPv6 relay agents may be configured to include a subscriber-ID option in relayed (RELAY-FORW) DHCPv6 messages. How the subscriberid is assigned and the mechanisms used to configure it are outside the scope of this memo.

4. DHCPv6 Server Behavior

This option provides additional information to the DHCPv6 server. The DHCPv6 server may use this information, if available, in addition to other relay agent option data, other options included in the DHCPv6 client messages, and physical network topology information in order to assign IP addresses, delegate prefixes, and/or other configuration parameters to the client. There is no special additional processing for this option.

There is no requirement that a server return this option and its data in a RELAY-REPLY message. 


\section{Security Considerations}

As the subscriber-id option is only exchanged between relay agents and DHCPv6 servers, [1], Section 21.1, provides details on securing DHCPv6 messages sent between servers and relay agents. [1], Section 23, provides general DHCPv6 security considerations.

6. IANA Considerations

IANA has assigned a DHCPv6 option code (38) for the Relay Agent Subscriber-ID Option.

\section{Acknowledgements}

Thanks to Richard Johnson, Theyn Palaniappan, and Mark Stapp as this document is essentially an edited version of their memo [3] .

8. References

8.1. Normative References

[1] Droms, R., Bound, J., Volz, B., Lemon, T., Perkins, C., and M. Carney, "Dynamic Host Configuration Protocol for IPv6 (DHCPv6)", RFC 3315, July 2003.

\subsection{Informative References}

[2] Troan, O. and R. Droms, "IPv6 Prefix Options for Dynamic Host Configuration Protocol (DHCP) version 6", RFC 3633, December 2003.

[3] Johnson, R., Palaniappan, T., and M. Stapp, "Subscriber-ID Suboption for the Dynamic Host Configuration Protocol (DHCP) Relay Agent Option", RFC 3993, March 2005.

[4] Postel, J. and J. Reynolds, "Telnet Protocol Specification", STD 8, RFC 854, May 1983.

[5] Droms, R., "Dynamic Host Configuration Protocol", RFC 2131, March 1997. 
Author's Address

Bernard Volz

Cisco Systems, Inc.

1414 Massachusetts Ave.

Boxborough, MA 01719

USA

Phone: +19789360382

EMail: volz@cisco.com 
Full Copyright statement

Copyright (C) The Internet Society (2006).

This document is subject to the rights, licenses and restrictions contained in BCP 78, and except as set forth therein, the authors retain all their rights.

This document and the information contained herein are provided on an "AS IS" basis and THE CONTRIBUTOR, THE ORGANIZATION HE/SHE REPRESENTS OR IS SPONSORED BY (IF ANY), THE INTERNET SOCIETY AND THE INTERNET ENGINEERING TASK FORCE DISCLAIM ALL WARRANTIES, EXPRESS OR IMPLIED, INCLUDING BUT NOT LIMITED TO ANY WARRANTY THAT THE USE OF THE INFORMATION HEREIN WILL NOT INERINGE ANY RIGHTS OR ANY IMPLIED WARRANTIES OF MERCHANTABILITY OR FITNESS FOR A PARTICULAR PURPOSE.

Intellectual Property

The IETF takes no position regarding the validity or scope of any Intellectual Property Rights or other rights that might be claimed to pertain to the implementation or use of the technology described in this document or the extent to which any license under such rights might or might not be available; nor does it represent that it has made any independent effort to identify any such rights. Information on the procedures with respect to rights in RFC documents can be found in BCP 78 and BCP 79 .

Copies of IPR disclosures made to the IETF Secretariat and any assurances of licenses to be made available, or the result of an attempt made to obtain a general license or permission for the use of such proprietary rights by implementers or users of this specification can be obtained from the IETF on-line IPR repository at http://www.ietf.org/ipr.

The IETF invites any interested party to bring to its attention any copyrights, patents or patent applications, or other proprietary rights that may cover technology that may be required to implement this standard. Please address the information to the IETF at ietf-ipreietf.org.

Acknowledgement

Funding for the RFC Editor function is provided by the IETF Administrative Support Activity (IASA). 